\title{
Prediction of Ground Vibrations Induced by Urban Railway Traffic: An Analysis of the Coupling Assumptions Between Vehicle, Track, Soil, and Buildings
}

\author{
Georges Kouroussis \\ Université de Mons - UMONS, Faculty of Engineering, Department of Theoretical Mechanics, Dynamics and \\ Vibrations, Place du Parc, 20 - 7000 Mons, Belgium
}

\section{Laurent Van Parys}

Université de Mons - UMONS, Faculty of Engineering, Department of Civil Engineering, Place du Parc, $20-$ 7000 Mons, Belgium

\section{Calogero Conti and Olivier Verlinden}

Université de Mons - UMONS, Faculty of Engineering, Department of Theoretical Mechanics, Dynamics and Vibrations, Place du Parc, 20 - 7000 Mons, Belgium

\begin{abstract}
(Received 16 August 2012; revised February and April of 2013; accepted 17 April 2013)
This paper is concerned with the problem of ground vibrations induced by railway traffic and its modelling through a decoupled approach, using only the finite element modelling for evaluating the ground waves propagation. The vehicle/track dynamics is calculated separately. An important modelling aspect is undoubtedly the track/soil interaction, which can play an important role in the generation of seismic waves. To avoid excessive computational resources, a coupled lumped mass model (CLM model) of the soil has been recently developed and is considered in this study. The influence of ballast and soil stiffnesses is presented, in order to confirm the range of validity of the CLM model. Combined with a discrete two layer model of the track, it offers the possibility of working with a simple compound track/soil model. A comprehensive analysis is provided to show the benefit of the finite element model with the proper radiation conditions at infinity, for analysing the structural response of a building located in the vicinity of the track. Focusing on typical results based on the tram of Brussels, the effects of track/soil and soil/structure coupling are investigated. Modal analyses of the vehicle and the building are presented in order to understand the effects of seismic wave amplification, especially when the source contains frequencies close to the natural frequencies of the building.
\end{abstract}

\section{INTRODUCTION}

Problems related to vibrations in buildings represent an important environmental issue in network designs, especially for nearby structures in densely populated cities. Ground vibrations induced by road and railway traffic can cause nuisance to occupants, malfunctioning of sensitive equipment (surgery or high-tech production), and even damage to buildings, if the vibration level exceeds critical values (e.g., $5 \mathrm{~mm} / \mathrm{s}$, according to DIN 4150 standards $^{1}$ ). The growing traffic volume, the higher population density, and the diminishing distance between the vehicle and the structure amplify the overall vibration nuisance. Although most of general traffic exposure vibrations (smooth surface) are not uncomfortable for people, the passing of heavy vehicles on a road or track with an uneven surface is often pointed out as the main vibratory nuisance felt by city residents.

Brussels, the capital of Belgium and of the European Union, is comprised of a heavily urban territory with over one million residents. The railway network in the Brussels Capital Region $\left(160 \mathrm{~km}^{2}\right)$ consists not only of the urban tramway network but also of the intercity and international train lines, since it rep- resents a vital link in the high speed train (HST) network in the northwest of Europe. Although railway transport appears as the most promising solution to traffic congestion, the development of new lines is confronted with the availability of convenient areas and the mistrust of the dwellers likely to be submitted to new nuisances. These difficulties have been largely encountered during the implementation of the new suburban light rail network. Presently, the tramway represents $25 \%$ of urban transport in Brussels.

The accurate prediction of railway-induced ground vibrations, and of the associated structural response, needs an understanding of the complex mechanisms of the generation and propagation of seismic vibration waves. The usefulness of a numerical prediction model has been well emphasized by various authors during the last decades. Empirical models, derived from in situ measurements, and commonly used at the very beginning, have been replaced by numerical models, which are more general and not limited to situations covered by the in situ measurements. Crispino and D'Apuzzo ${ }^{2}$ recently described the measurement of road traffic-induced vibrations on a heritage building in Naples, which conducted an empirical 